\title{
Theoretical Analysis and Experimental Validation of Single-Phase Direct vs. Cascade Voltage Control in Islanded Microgrids
}

\author{
T.L. Vandoorn, Member, IEEE, C.M. Ionescu, Member, IEEE, J.D.M. De Kooning, Member, IEEE, R. De Keyser \\ and L. Vandevelde, Senior Member, IEEE
}

\begin{abstract}
The increasing number of distributed generation units has led to the development of microgrids, to which the distributed generators are commonly interfaced by means of a voltage-source inverter (VSI). When the microgrid is operating independently of the power system, i.e. in islanded mode, two levels of control can be distinguished for these VSIs: power control and voltage control (frequency and amplitude). The set-point values for the voltage controller are obtained from the power controller. This paper investigates theoretically and experimentally the benefits of using several PID control structures for the voltage control. Theoretical insights into the dynamics of such a system emphasize the benefits of measuring current signals for control purposes and adding voltage measurements to the output of the controllers. Direct voltage control and cascade voltage control are compared both with and without forward compensation of the grid voltage. Simulation and experimental results are given showing that such PID-type controllers on a digital signal processor are simple yet effective strategies for an accurate voltage control in islanded microgrids.
\end{abstract}

Index Terms-Cascade control, distributed generation, microgrids, PID control, voltage control, voltage-source inverter

\begin{tabular}{ll} 
& \multicolumn{1}{c}{ NomenClATURE } \\
$C_{\mathrm{dc}}$ & Dc-link capacitance $(\mathrm{F})$ \\
$C$ & Filter capacitance $(\mathrm{F})$ \\
DER & Distributed Energy Resources \\
$\mathrm{DG}$ & Distributed generation \\
$f$ & Grid frequency $(\mathrm{Hz})$ \\
$L$ & Filter inductance $(\mathrm{H})$ \\
'nom' & Nominal/rated value \\
$P$ & Ac-side power $(\mathrm{W})$ \\
$P_{\mathrm{dc}}$ & Dc-side input power $(\mathrm{W})$ \\
$V_{\mathrm{dc}}$ & Dc-link voltage $(\mathrm{V})$ \\
VSI & Voltage-source inverter
\end{tabular}

Manuscript received November 30, 2011. Accepted for publication June 15,2012

Copyright (c) 2009 IEEE. Personal use of this material is permitted However, permission to use this material for any other purposes must be obtained from the IEEE by sending a request to pubs-permissions@ieee.org.

T.L. Vandoorn, J.D.M. De Kooning and L. Vandevelde are with the Department of Electrical energy, Systems and Automation, Research group EELAB - Electrical Energy Laboratory, Ghent University, Sint-Pietersnieuwstraat 41, B-9000 Ghent, Belgium, e-mail: Tine.Vandoorn@UGent.be.

R. De Keyser and C.M. Ionescu are with the Department of Electrical energy, Systems and Automation, Research group SYSTeMS, Ghent University, Technologiepark 913, B-9052 Ghent, Belgium.

The research was carried out in the frame of the Inter-university Attraction Poles programme IAP-VII-43, funded by the Belgian Government. The work of T. Vandoorn is financially supported by a Ph. D. fellowship of the Research Foundation - Flanders (FWO). The research of J. D. M. De Kooning is funded by the Special Research Fund (BOF) of Ghent University (Belgium). C.M. Ionescu is a post-doctoral researcher of FWO.

\section{INTRODUCTION}

$\mathbf{N}$ EW opportunities for the coordinated operation of distributed energy resources (DER) arise with the introduction of the microgrid [1], [2]. The Consortium for Electric Reliability Technology Solutions presents a microgrid as a system providing both power and heat, consisting of an interconnected network of generators, loads and energy storage elements [1]. Generally, these microgrids consist of a high share of renewable energy based DG units of which the intermittent nature poses further challenges on the microgrid control. These networks are typically connected to the distribution grid via a single point of connection. Microgrids can enhance local reliability, reduce feeder losses, support local voltage, increase efficiency through combined heat and power and provide uninterruptible power supply functions [3]. Furthermore, it is expected that the network will become more intelligent based on a gradual evolutionary change of the electrical system, with microgrids playing a key role in this evolution to a smart grid [4], [5]. Some of the main features of a smarter grid are active network management, automatic metering infrastructure, bidirectional communication, further development of the energy market and extended demand response [6], [7]. The European Commission is highlighting the importance of smart grids, e.g. in the Strategy Research Agenda (SRA) [8]. In this context, the European Energy Research Alliance (EERA) is founded to support the European Strategic Energy Technology (SET) plan. One of its programs is the smart grids program [9].

The intended operation of the microgrid can follow two operating conditions: grid-connected mode and islanded mode. In the grid-connected mode, the DER in the microgrid support the main grid. In this configuration, the transmission network mainly determines the voltage of the system and the microgrid can exchange power with the main grid. The microgrid can be disconnected from the main grid during large disturbances (voltage collapse, faults, poor power quality), feeding only local loads in the islanded mode. Islanded microgrids can also be applied in remote places where no main grid is available. In this configuration, the microgrid elements are responsible for voltage control (frequency and amplitude) and power control, unlike in the grid-connected mode. Generally, in the islanded operating mode, droop control [10]-[13] and its variants [14][21] are applied, all avoiding inter-unit communication for a robust operation of the microgrid. These droop control algorithms enable the balancing and sharing of the active and 
reactive power. Microgrids are being tested and demonstrated in many projects around the world such as the E.U. Microgrids and More Microgrids projects, the U.S. CERTS microgrid and the NEDO microgrid projects in Japan [1], [22]-[24].

Most DER are connected to the ac grid through powerelectronic interfaces, mostly voltage-source inverters (VSIs). With the increased emergence of inverter-based microgrids, the control of these VSIs has become an important concern for the grid operation [25]. For inverters in the grid-connected operating condition, the microgrid voltage is imposed by the main power system. Mostly, a current-controlled inverter is used, e.g., current-controlled photovoltaic systems with maximum power point tracking [26]. However, for inverters in islanded microgrids, the voltage is not determined by the power system. Therefore, the voltage control is a key issue in islanded microgrids. Several control strategies such as PID control [17], sliding mode control [27] and fuzzy logic control [28] can be applied. Due to their limited number of parameters and straightforward implementation in practice, PID controllers and variants on PID control are used in a wide range of applications [29]-[31], including converter control [18], [32].

The original contribution of this paper stands within the theoretical insight into the system envisaged for control purposes, completed by experimental validation. The efficient use of all available signals (i.e. voltage and current) at different locations in the system is a key issue addressed in this paper. For this, direct control and cascade control are compared. Only cascade control uses grid current measurements for the voltage control. Next to current measurements, also the effect of adding grid voltage measurements to the output of the controllers is studied. As a result of the theoretical analysis, simple yet effective control strategies arise as intrinsic solutions to the problem. It is shown that for the cascade control, a P controller in series with PI control is sufficient, while direct control requires the usage of a PID controller.

The paper is organized as follows. First, a theoretical analysis of voltage control in an islanded microgrid is given. Second, direct control and cascade control are compared in simulation. Also, the effect of forward voltage compensation is studied. Finally, the theoretical findings and the conclusions from the simulations are validated by means of experiments on a full-bridge IGBT converter controlled by a digital signal processor.

\section{TheOretiCAL ANALYsis}

\section{A. Circuit Analysis}

The physical system used to develop the theoretical analysis and perform experimental tests is illustrated in Fig. 11. The differential equations describing the system are given by:

$$
\begin{aligned}
& L \frac{d i_{\mathrm{L}}(\mathrm{t})}{d t}=v_{\mathrm{s}}(\mathrm{t})-v_{\mathrm{g}}(\mathrm{t}), \\
& C \frac{d v_{\mathrm{g}}(\mathrm{t})}{d t}=i_{\mathrm{L}}(\mathrm{t})-i_{\mathrm{g}}(\mathrm{t}) .
\end{aligned}
$$

When averaged over one switching period, the switch voltage $v_{\mathrm{s}}$ can be written in terms of the dc bus voltage $v_{\mathrm{dc}}$ by introducing the duty ratio $\delta$ of the switches $(\delta \in[-1,1])$ :

$$
v_{\mathrm{s}}(\mathrm{t})=\delta(\mathrm{t}) v_{\mathrm{dc}}(\mathrm{t}) .
$$

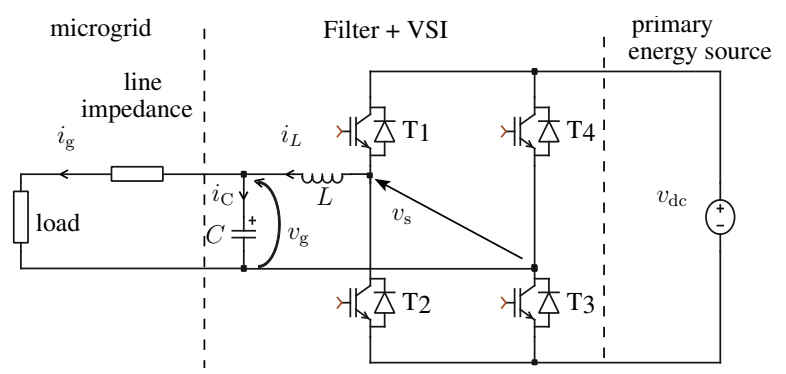

Fig. 1. VSI interface between the microgrid and an energy source. The VSI needs to control the microgrid voltage $v_{\mathrm{g}}$.

The load is considered as a black-box, thus, unknown and variable. For the tuning of the controllers $v_{\mathrm{dc}}(\mathrm{t})$ is considered as a constant parameter, $V_{\mathrm{dc}}$. The differential equations are transformed to the Laplace domain:

$$
\begin{aligned}
& s L i_{\mathrm{L}}(\mathrm{s})=v_{\mathrm{s}}(\mathrm{s})-v_{\mathrm{g}}(\mathrm{s}), \\
& s C v_{\mathrm{g}}(\mathrm{s})=i_{\mathrm{L}}(\mathrm{s})-i_{\mathrm{g}}(\mathrm{s}) .
\end{aligned}
$$

For the purpose of control of the grid voltage $v_{\mathrm{g}}$, the source voltage $v_{\mathrm{s}}$ needs to be manipulated. However, the system has inherent feedback loops. Hence, in the context of providing good control performance of microgrids, irrespective of load disturbances and delivering a specified amplitude and frequency, it is necessary to understand the dynamics of such a system. In the remainder of this section, several aspects are presented, structured in two main parts: without, and with the grid current $i_{\mathrm{g}}$ available as a measured signal.

\section{B. Considering the current $i_{\mathrm{g}}$ not available for control}

The general scheme equivalent to the model from (4)-(5) is that of the LC filter illustrated in Fig. 2.

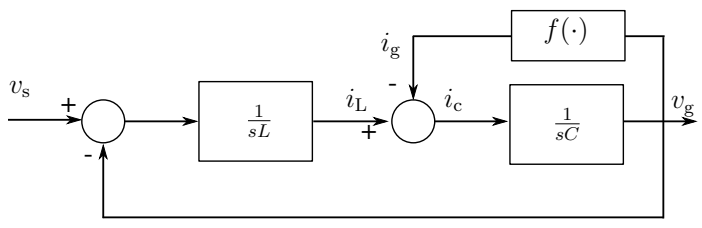

Fig. 2. Considered system: dynamics of the LC filter

In practice, $i_{\mathrm{g}}$ is varying, posing challenges for the controller. This signal represents the load from the consumer, which is a stochastic, unknown disturbance in the considered system $\left(i_{\mathrm{g}}\right.$ is a variable, unknown function $f(\cdot)$ of $\left.v_{\mathrm{g}}\right)$. If this is an independent disturbance, then classical feedback control strategies may easily be applied. However, if such a control is applied blindly, it may lead to sub-optimal results, since the assumption that $i_{\mathrm{g}}$ is an independent variable is false. The reason for this is the presence of the internal feedback loop as a function of the grid voltage. Let us consider for simplicity that the current $i_{\mathrm{g}}$ is related to the grid voltage by a varying 
load which can be represented in its simplest form by a resistor $R\left(f(\cdot)=R^{-1}\right)$. This leads to the scheme in Fig. 3

The question that now arises is whether or not measuring the current $i_{\mathrm{L}}$ would be useful for the control purposes. For this analysis, the equivalent loop transfer functions for the circuit from Fig. 3 are derived, with $\tau_{c}=R C$.

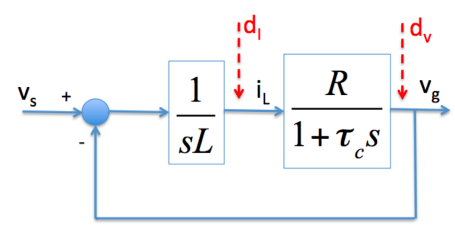

Fig. 3. Equivalent scheme of the LC filter, with inherent feedback from $i_{\mathrm{g}}$ (through a resistor $R$ ) included in the loop. Notice two disturbance inputs $d_{\mathrm{I}}$ and $d_{\mathrm{V}}$.

The transfer function from the manipulated variable to the output is given by:

$$
\frac{v_{\mathrm{g}}}{v_{\mathrm{s}}}=\frac{\frac{R}{s L\left(1+\tau_{c} s\right)}}{1+\frac{R}{s L\left(1+\tau_{c} s\right)}}=\frac{1}{1+\tau_{L} s\left(1+\tau_{c} s\right)},
$$

with $\tau_{L}=\frac{L}{R}\left(\tau_{c}\right.$ and $\tau_{L}$ are both varying with the load $R$ ). The transfer function from the disturbance $d_{I}$ to the output is given by:

$$
\frac{v_{\mathrm{g}}}{d_{\mathrm{I}}}=\frac{\frac{R}{1+\tau_{c} s}}{1+\frac{R}{s L\left(1+\tau_{c} s\right)}}=\frac{L s}{1+\tau_{L} s\left(1+\tau_{c} s\right)}
$$

Based on these transfer functions, the scheme from Fig. 3 can be re-arranged as in Fig. 4

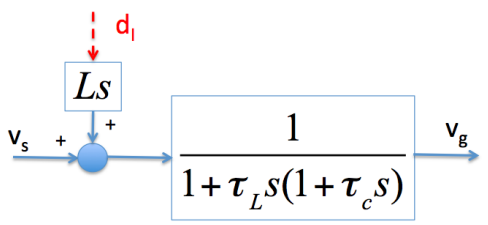

Fig. 4. Equivalent scheme of the LC filter, based on the derived transfer functions.

The combination of Figs. 3 and 4 leads to the block scheme depicted in Fig. 5 of the system to be controlled, where the current $i_{\mathrm{L}}$ appears explicitly in the loop. Compared to Fig. 3 . Fig. 5 has no internal feedback loop.

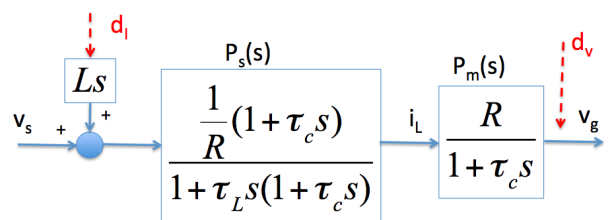

Fig. 5. Equivalent scheme of the LC filter, including $i_{\mathrm{L}}$ as an explicit signal in the loop.

Now it is easy to see from this representation, that cascade control could be applied if the signal $i_{\mathrm{L}}$ is available as a measurement. $P_{m}(s)$ denotes the transfer function of the system to be controlled by a master controller; $P_{s}(s)$ denotes the transfer function to be controlled by a slave controller. Notice that in order to follow set-points and to reject input disturbances, the master controller should have an integral action, while for the slave controller a gain is sufficient [33].

At this moment, it is necessary to verify whether cascade control would be useful. The speed of response of the two transfer functions $P_{m}(s)$ and $P_{s}(s)$ is determined by the locations of the poles. The pole of the master transfer function is given by $s=-\frac{1}{\tau_{c}}$. For the two poles of the slave transfer function, the root locus analysis [33] is applied to the characteristic equation $1+\frac{1}{\tau_{c} \tau_{L}} \frac{1}{s\left(s+\frac{1}{\tau_{c}}\right)}$. This is depicted in Fig. 6 (left). It follows that the location depends on the value of $1 / L C$, but the two poles are always located to the right of $s=-\frac{1}{\tau_{c}}$. This implies that $P_{s}(s)$ is slower than $P_{m}(s)$.
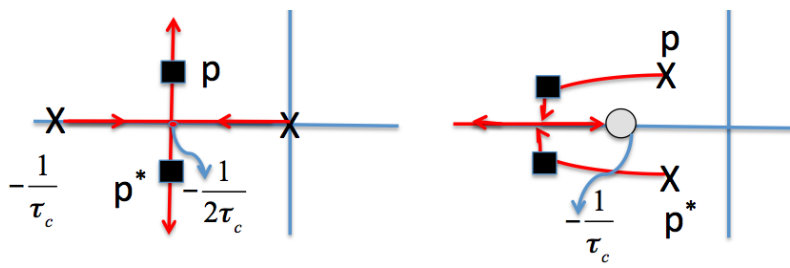

Fig. 6. Left: Root locus for the open loop $P_{s}(s)$. Right: Root locus for the closed loop of the slave control, with a gain controller.

Consider now the case when $i_{\mathrm{L}}$ is measured and used in a slave feedback loop, e.g. using a simple $\mathrm{P}$-controller with gain $K_{s}$. This feedback loop is given by the transfer function

$$
\frac{K_{s} P_{s}(\mathrm{~s})}{1+K_{s} P_{s}(\mathrm{~s})} \text {. }
$$

The denominator can be rewritten as:

$$
1+K \frac{\left(s+1 / \tau_{c}\right)}{(s-p)\left(s-p^{*}\right)},
$$

with $p$ and $p^{*}$ the poles of the open loop $P_{s}(\mathrm{~s})$ and $K$ the loop gain. After applying root locus analysis to this characteristic equation, the obtained location of the poles is depicted in Fig. 6 (right). The conclusions from this analysis are twofold: i) that the poles of this slave loop $(8)$ can be placed to the left of $s=-\frac{1}{\tau_{c}}$, for suitable values of the gain $K$; and ii) that the zero from the slave loop (8) cancels the pole of the master transfer function $P_{m}(s)$. Hence, the system with slave loop is always faster than the open loop transfer function in Fig. 5 and cascade control is thus useful.

It can be concluded that if the current $i_{g}$ is not available for control, preferably, the current $i_{\mathrm{L}}$ should be used in a slave loop with a P-controller. Also, $v_{\mathrm{g}}$ should be used in the master PI control loop.

\section{Considering the current $i_{\mathrm{g}}$ available for control}

If $i_{\mathrm{g}}$ could be measured, then (hypothetically) one can compensate for the feedback from $v_{\mathrm{g}}$ in the initial scheme from Fig. 2 by injecting at the same point where $i_{g}$ enters the loop, an $i_{\mathrm{g}}$ signal with an opposite sign. Of course, this is not physically possible; however, based on the theoretical 
developments hereafter, a practical solution will be given (ref. section II-C4). Assuming that the ideal case of perfect compensation would be possible, the equivalent basic scheme will be the same as in Fig. 2, but with the signal $i_{\mathrm{g}}$ removed. In this case, a significant observation can be made: the derivative of $v_{\mathrm{g}}$ is $i_{\mathrm{L}}$ (it will be shown further how this observation can be used):

$$
\frac{v_{\mathrm{g}}}{i_{\mathrm{L}}}=\frac{1}{s C} .
$$

1) Direct Control: If only voltage control would be used, it follows that the transfer function of the system becomes:

$$
\frac{v_{\mathrm{g}}(s)}{v_{\mathrm{s}}(s)}=\frac{1}{1+L C s^{2}},
$$

with the corresponding scheme given in Fig. 7, showing the inherent feedback in the system. The corresponding root locus of the closed loop if a PI master controller is used, is given in Fig. 8 (left). It follows that a PI-controller cannot stabilize the system, hence derivative action is absolutely necessary. The corresponding root locus of the closed loop if a PID-control is used, is given in Fig. 8 (right). In this case, a stable closed loop is obtained, at the expense of increased noise effects (due to differentiation) and necessity of adding noise filters in the physical system.

2) Cascade Control: At this point, it becomes interesting to consider the benefits of adding a slave loop. Recall here the observation from (10), which implies that an inner loop can be introduced (current loop) to replace the explicit derivative action in the PID controller. This is based on the fact that $i_{L}$ is proportional to the derivative of $v_{g}$, ref (10). Using an inner loop with feedback from $i_{L}$ is thus equivalent to using Daction in the master PID controller. However, it does not have the mentioned noise sensitivity. The scheme of the closed loop becomes that of Fig. 9 , with $P$ the proportional controller of the inner loop and a PI controller in the outer loop.

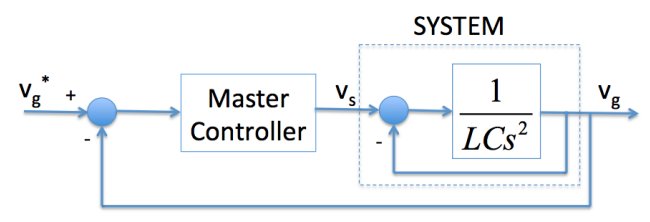

Fig. 7. Direct control: closed loop scheme for voltage control, when $i_{\mathrm{g}}$ in Fig. 2 is compensated.

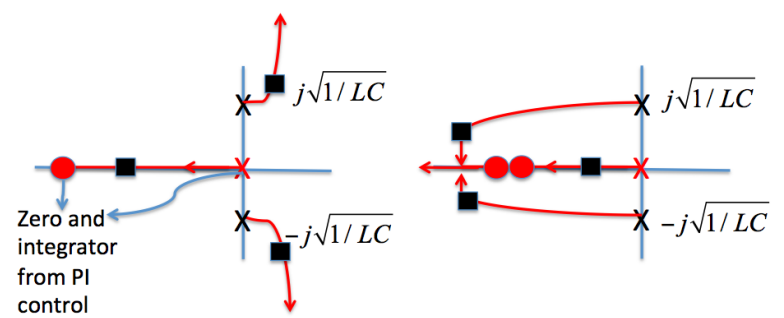

Fig. 8. Left: Root locus for the closed loop with a PI-controller. Right: Root locus for the closed loop with a PID-controller.

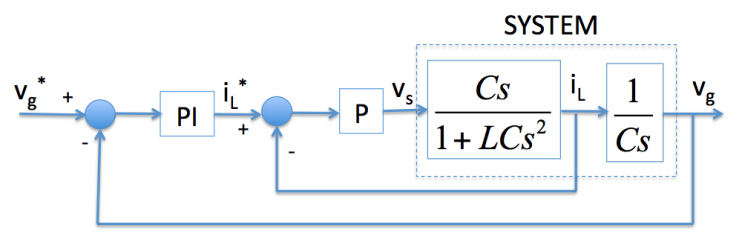

Fig. 9. Cascade closed loop control scheme with inherent derivative action from the current loop on $i_{\mathrm{L}}$.

3) Feedforward from $v_{g}$ : Starting again from Fig. 2, and similarly to injecting $i_{\mathrm{g}}$ into the system, a measurement of $v_{\mathrm{g}}$ can be injected to the output of the P-controller in the inner loop as illustrated in Fig. 10 Notice that, opposed to the former case, this is physically possible since the controller is software-implemented and injecting $v_{\mathrm{g}}$ is just an additional operation. The effect of adding $v_{\mathrm{g}}$ will compensate for the physically present feedback from the $v_{\mathrm{g}}$ signal (ref. Fig. 10 p.

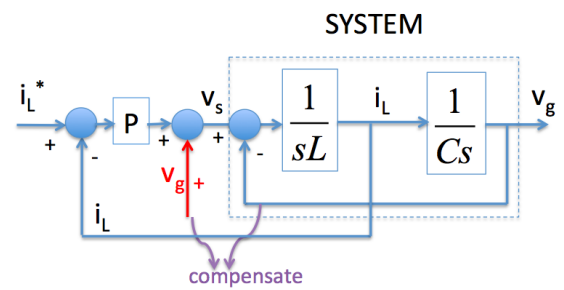

Fig. 10. Schematic representation of injecting $v_{\mathrm{g}}$ in the inner current loop.

4) Practical solution to inject $i_{g}$ : In the ideal case of perfect models and perfect measurements and assuming a Pcontroller with a gain $K$ sufficiently large, the loop from $i_{\mathrm{L}}^{*}$ to $i_{\mathrm{L}}$ is approximately equal to 1 , i.e. its transfer function $\frac{1}{1+L s / K} \cong 1$. This implies that $i_{\mathrm{L}} \cong i_{\mathrm{L}}^{*}$, which immediately suggests the physical solution to inject $i_{g}$ at the output of the master controller (a PI-controller), leading to the final configuration depicted in Fig. 11. In this way, internal feedback of $i_{\mathrm{g}}$ is compensated for.

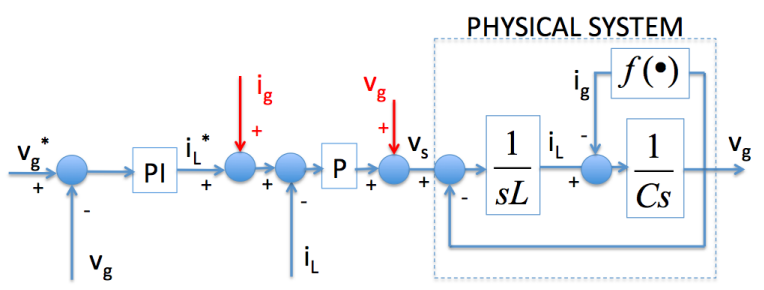

Fig. 11. Final configuration with practical solution for injecting $i_{g}$ and $v_{g}$ into the closed loop control scheme.

To summarize, the theoretical analysis suggests that i) cascade control from $i_{\mathrm{L}}$ is always useful and ii) compensating for the signals $v_{\mathrm{g}}$ and $i_{\mathrm{g}}$ is beneficial for the overall control performance.

5) Conclusion: If the current $i_{g}$ is available for control, the best control structure is again a master-slave configuration with a PI master controller and a P slave controller. However, compared to the situation where $i_{\mathrm{g}}$ is not used (section II-B), 
there now is an advantage which is extremely important from practical point of view: thanks to the $i_{\mathrm{g}}$ injection, the tuning of the controllers became independent of the load (R). This is a significant benefit from control point of view, since in practice the load is varying and not known.

\section{COMPARISON OF CONTROLLERS}

In this paper, single-phase voltage control is studied. The control of the VSI is usually obtained in the rotating $d q$ reference frame synchronous to the grid voltage, for example in [34]. A disadvantage of this method is the numerical complexity, because of, for example, the need for a harmonic reference. By using the Clarke and Park transformations, the quantities in a three-phase balanced sinusoidal system in steady state are transformed into dc-Park components, which is an advantage for control issues. However, in three-phase asymmetrical systems or in systems with voltage harmonics, the Park transformation does not result in dc-quantities. In single-phase systems, the standard Park or Clarke transformations are even not applicable. In the current paper, the control is performed in the time domain without transformation of reference frame and by using conventional PI-regulators. The extension to a three-phase grid can follow an analogous PI(D) control strategy, but is out of the scope of this paper. In this case, the phase shift between the three phases of the electrical system should be considered or frame transformation can be used as is often the case in literature.

As suggested in the previous section, direct control implies the necessity of a PID controller while for cascade control, a PI-P combination is sufficient. Hence, direct and cascade control, with the suggested PID-type controllers are compared. In cascade control, the available current measurements $i_{\mathrm{g}}$ and $i_{\mathrm{L}}$ are used in the control scheme, which is not the case in direct control. The available measurement of $v_{\mathrm{g}}$ can be used in the control scheme as well. Forward voltage compensation is achieved in Fig. 11 by adding $v_{\mathrm{g}}$ to the output of the slave controller. In the direct control scheme, $v_{\mathrm{g}}$ can also be added to the output of the PID controller. Hence, the cases with and without adding $v_{\mathrm{g}}$ to the output of the controller (forward voltage compensation) are compared for the direct as well as the cascade control structure.

The simulations are performed upto the level of the converter switches which are arranged in a full-bridge configuration. For the tuning of the controllers, per unit voltages and currents are used with reference values of $400 \mathrm{~V}$ and 10 A respectively. The dc-link voltage equals $400 \mathrm{~V}$, the filter inductance $L$ equals $2.2 \mathrm{mH}$ and filter capacitance $C$ is $5 \mu \mathrm{F}$. The per unit reference value equals the dc-link voltage. This results in a convenient implementation of the forward voltage compensation. The reason is that in the averaged model of the VSI with pulse-width modulation, $v_{s}$ equals $\delta v_{\mathrm{dc}}$, with $v_{\mathrm{dc}}=1$, hence $\delta$ is easily obtained. For practical reasons in the experimental part, a $25 \mathrm{~Hz}$ network is considered. Both the sampling frequency and switching frequency equal $10 \mathrm{kHz}$. The reference voltage for the per unit calculation in the figures equals $230 \sqrt{2} \mathrm{~V}$ and the reference current is $20 \mathrm{~A}$.

The following reference loads are used:
- Firstly, the set point voltage equals $230 \mathrm{~V}$ rms. At $t=$ $30 \mathrm{~ms}$ this set point value drops to $195 \mathrm{~V}$ and is restored at $t=40 \mathrm{~ms}$. A resistive load of $65 \Omega$ and an RL load of $65 \Omega$ in series with $310 \mathrm{mH}$, thus with $\cos \phi=0.8$ are present. The former load turns off at $t=50 \mathrm{~ms}$ and the last one at $t=70 \mathrm{~ms}$.

- Secondly, the values of $C$ and $L$ are doubled compared to the values that the controllers are tuned for. In this way, parameter sensitivity to model faults can be studied. Next, after $0.5 \mathrm{~s}$, the robustness to measurement noise is studied by including band-limited white noise in the measurements of $i_{\mathrm{g}}, i_{\mathrm{L}}$ and $v_{\mathrm{g}}$ varying between $-5 \%$ and $+5 \%$. This $5 \%$ value is a high value, in practice, a lower measurement error can be assumed. In the simulation plots, the measurements, thus including this noise, are depicted.

The controllers are tuned based on the phase margin (30$60^{\circ}$ ) and bandwidth. The bandwidths of the direct controller and the slave controller in cascade control should be higher than $1 \mathrm{kHz}$ (the 40-th harmonic in the considered network) and the voltage controller in cascade control should be slower. First, the slave loop is tuned, next the master loop. An advantage is that the transfer function of the system is wellknown, only depending on the values of $L$ and $C$. The tuning can be done either manually (analytically), either using computer-aided-design tools (the root locus tool in Matlab is used in this paper). Details of the controllers are given below.

\section{A. Direct control}

The grid voltage $v_{\mathrm{g}}(\mathrm{t})$ is controlled to its reference value $v_{\mathrm{g}}^{\star}(\mathrm{t})$. As shown in $\$$ III, a PID controller is required for this purpose when using direct control.

1) Without forward voltage compensation: For the direct control without adding $v_{\mathrm{g}}$ to the output of the controller (forward voltage compensation), the following PID controller is tuned:

$$
C(z)=0.24573 \frac{z^{2}-2.2 z+2.066}{z(z-1)} .
$$

With this controller, the control circuit has the following characteristics: settling time $T_{s}=1.84 \mathrm{~ms}$, overshoot $\% \mathrm{OS}=$ 25.8, phase margin $\mathrm{PM}=48.8^{\circ}$, gain margin $\mathrm{GM}=6.33 \mathrm{~dB}$ and open loop bandwidth $f_{b}=1.71 \mathrm{kHz}$. From the Bode plot, it follows that the phase lag for $25 \mathrm{~Hz}$ (which is the frequency of the reference signal) equals $4.2^{\circ}$. This is compensated by including a phase-lead in the reference signal. This controller is referred to as PID 1.

For the first reference load, a good voltage tracking is obtained according to the regime results, e.g., from $45<t<50 \mathrm{~s}$ shown in Fig. $12(a, b)$. The transient after the load switching shows some oscillations. That the phase-lag compensation significantly affects the controller performance, is shown in Fig. 12 (c,d), in this case, the phase-lag compensation of $4.2^{\circ}$ is omitted. This leads to a larger regime error. Hence, an accurate phase-lag calculation is required.

The PID 1 controller does not obtain a stable operation with the second reference load. A stable operation is however obtained when $L$ remains unchanged as depicted in 


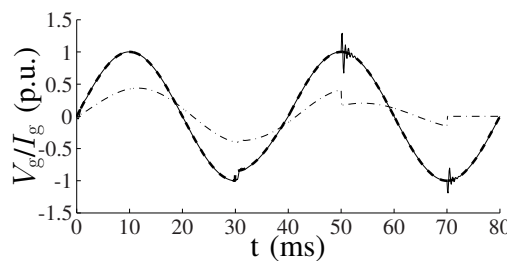

(a) First reference load, with phase-lag compensation: $V_{\mathrm{g}}(\mathrm{t})$ and $I_{\mathrm{g}}(\mathrm{t})$

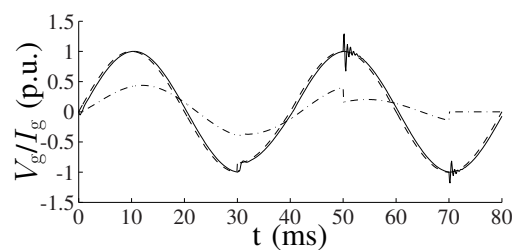

(c) First reference load, without phase-lag compensation: $V_{\mathrm{g}}(\mathrm{t})$ and $I_{\mathrm{g}}(\mathrm{t})$

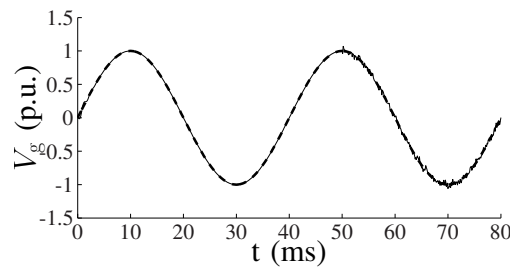

(e) Adapted second reference load, with phase-lag compensation: $V_{\mathrm{g}}(\mathrm{t})$

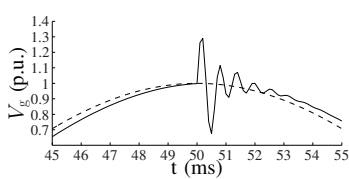

(d) Detail of $V_{\mathrm{g}}$ in (c)

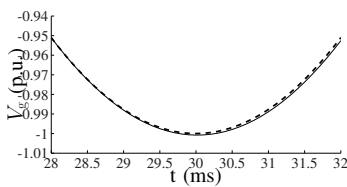

(f) Detail of $V_{\mathrm{g}}$ in (e)

Fig. 12. PID 1 - Direct control without forward compensation $\left(-=v_{\mathrm{g}}(\mathrm{t})\right.$ (p.u.); ---- $=v_{\mathrm{g}}^{\star}(\mathrm{t})$ (p.u.); -.-.-. $=i_{\mathrm{g}}(\mathrm{t})$ (p.u.))

Fig. 12(e,f), hence this controller is sensitive to $L$. The results from $t>50 \mathrm{~ms}$ show that the controller is sufficiently robust for measurement error.

For PID 1, an important difficulty is that accurate phaselag calculation is required, depending on an accurate system model. To overcome this issue, a variant of the PID 1 controller uses the same PID controller as the previous case, but with adding the set point voltage $v_{\mathrm{g}}^{\star}$ to its output. This controller is referred to as PID 2. The phase-lag compensation in the reference signal is omitted in PID 2 and the control scheme is depicted in Fig. 13. This leads to a more robust control design.

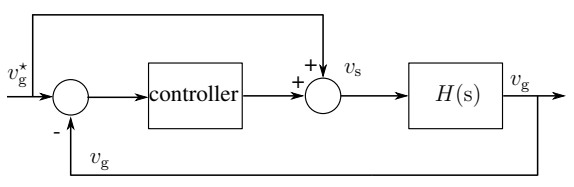

Fig. 13. PID 2: Direct control with adding $v_{\mathrm{g}}^{\star}$ to the output of the PID controller

The simulation results in case of the first reference load are depicted in Fig. 14. Similar steady-state voltage tracking and transient results are obtained as in case of the direct control without $v_{\mathrm{g}}^{\star}$ compensation and with phase-lag compensation.

Like the previous controller, this controller does not obtain a stable operation with the second reference load. When $L$ remains unchanged, similar results as in Fig. 12(e,f) are

obtained.

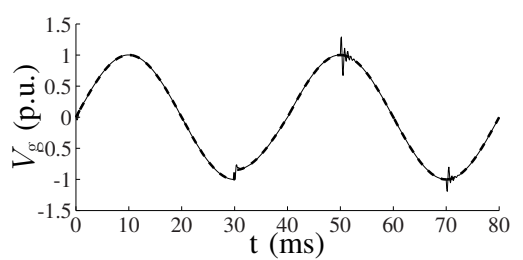

(a) First reference load: $V_{\mathrm{g}}(\mathrm{t})$ and $I_{\mathrm{g}}(\mathrm{t})$

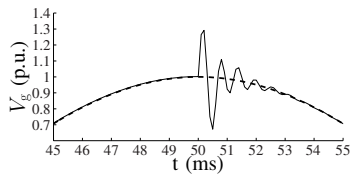

(b) Detail of $V_{\mathrm{g}}$ in (a)
Fig. 14. PID 2 - Direct control with adding $v_{\mathrm{g}}^{\star}$ to the output of the PID controller $\left(-=v_{\mathrm{g}}(\mathrm{t})\right.$ (p.u.); ---- $=v_{\mathrm{g}}^{\star}(\mathrm{t})$ (p.u. $\left.)\right)$

2) With forward voltage compensation: Second, forward voltage compensation is implemented by adding the measured voltage $v_{\mathrm{g}}$ to the output of the controller as depicted in Fig. 15. A new PID controller is tuned because the system changes from $H$ to $\frac{H}{1-H}$ due to this forward compensation. The following PID controller is tuned:

$$
C(z)=0.67432 \frac{(z-0.868)(z-0.3)}{z(z-1)} .
$$

With this controller, the control circuit has the following characteristics: $T_{s}=1.76 \mathrm{~ms}, \% \mathrm{OS}=46.5, \mathrm{PM}=40^{\circ}$, $\mathrm{GM}=6 \mathrm{~dB}, f_{b}=776 \mathrm{~Hz}$. From the Bode plot, it follows that the phase for $25 \mathrm{~Hz}$ (which is the frequency of the reference signal) equals $0.115^{\circ}$. This small value is thus not compensated by including a phase-lead in the reference signal. This controller is referred to as PID 3.

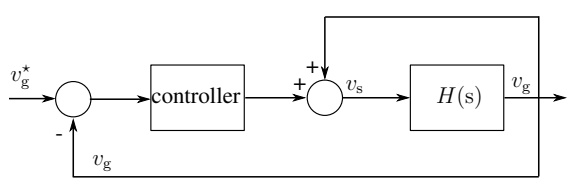

Fig. 15. PID 3: Direct control with forward compensation of $v_{\mathrm{g}}$

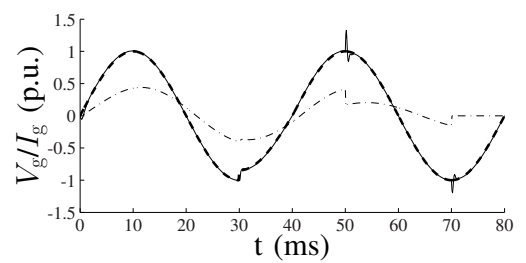

(a) First reference load: $V_{\mathrm{g}}(\mathrm{t})$ and $I_{\mathrm{g}}(\mathrm{t})$

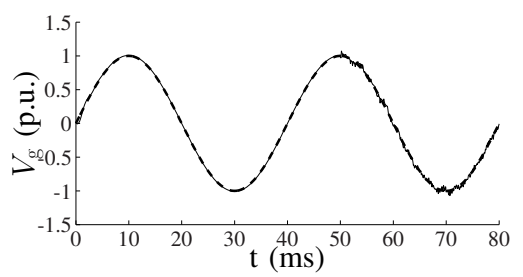

(c) Second reference load: $V_{\mathrm{g}}(\mathrm{t})$

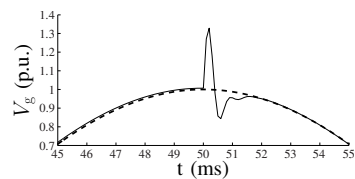

(b) Detail of $V_{\mathrm{g}}$ in (a)

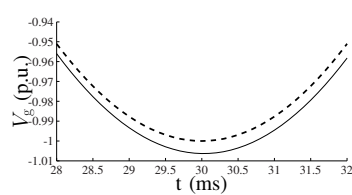

(d) Detail of $V_{\mathrm{g}}$ in (c)
Fig. 16. PID 3 - Direct control with forward compensation of $v_{\mathrm{g}}(-=$ $v_{\mathrm{g}}(\mathrm{t})($ p.u. $) ;-\cdot-=v_{\mathrm{g}}^{\star}(\mathrm{t})($ p.u. $) ;-\cdot-\cdot-=i_{\mathrm{g}}(\mathrm{t})($ p.u. $\left.)\right)$ 
In case of the first reference load in Fig. 16(a,b), a slightly less accurate steady-state tracking is obtained, but PID 3 results in a significant improvement in transient behavior compared to the previous case. This slightly less accurate regime tracking can be due to the lower bandwidth of this controller. For the second load in Fig. 16(c,d), the PID 3 controller outperforms PID 1 and PID 2, by reaching a stable operation despite the model faults in both $L$ and $C$.

\section{B. Cascade control}

As discussed in $\$$ III the usage of cascade control is possible in the considered system. Including current measurements that are not present in the direct control, is beneficial for the overall controller performance. In this paragraph, these two conclusions are investigated in simulation and the effect of extra voltage measurement (forward voltage compensation) is studied as well.

1) Without forward voltage compensation: For cascade control without adding $v_{\mathrm{g}}$ to the output of the controller (forward voltage compensation) as suggested in Fig. 9, theoretically, a $\mathrm{P}$ controller is sufficient in the current control loop. However, to obtain a sufficient phase margin, a PD controller with a small $\mathrm{D}$ action is used in the following simulations:

$$
C(z)=0.3455 \frac{z-0.22}{z},
$$

with this controller, the current control loop has the following characteristics: $\mathrm{PM}=30.1^{\circ}, \mathrm{GM}=7.78 \mathrm{~dB}, f_{b}=2.01 \mathrm{kHz}$ (open loop bandwidth). For the voltage controller, the following PI controller is tuned:

$$
C(z)=0.35134 \frac{z+2}{z-1} .
$$

With this controller, the voltage control loop has the following characteristics: $T_{s}=1.22 \mathrm{~ms}, \mathrm{PM}=63.5^{\circ}, \mathrm{GM}=6 \mathrm{~dB}, \% \mathrm{OS}=$ 10.4 and $f_{b}=497 \mathrm{kHz}$ (open loop bandwidth). From the Bode plot, it follows that the phase lag for $25 \mathrm{~Hz}$ (which is the frequency of the reference signal) equals $-3.03^{\circ}$, which is compensated by a phase-lead in the reference voltage. This controller is referred to as PIPD 1.

The simulation results in case of the first load are depicted in Fig. 17(a,b). An accurate steady-state tracking is obtained, analogous to the PID 1 and PID 2 control strategies and better than for the PID 3 option. For the second load in Fig. 17 ( c,d), a good robustness to model faults and noise are obtained.

Analogous as in the previous paragraph, PIPD 2 consists of the same PI-PD controller as the previous case but with addition of the set point voltage $v_{\mathrm{g}}^{\star}$ in the output of the controller to avoid the phase-lag compensation. PIPD 1 and PIPD 2 have similar results, analogous as in direct control with PID 1 and PID 2.

2) With forward voltage compensation: Second, a forward compensation of the measured voltage $v_{\mathrm{g}}$ is included according to Fig. 11. For this, a PI-P control strategy is sufficient. The $\mathrm{P}$ controller of the inner current control loop equals 0.35811 giving this loop a $f_{b}=1 \mathrm{kHz}$ and $\mathrm{PM}=53.9^{\circ}$. The PI

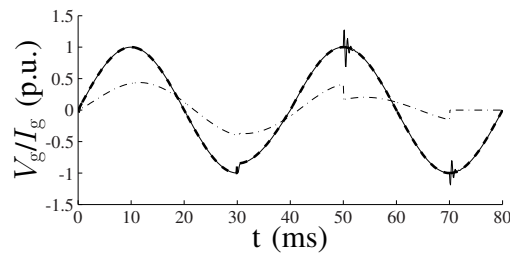

(a) First reference load: $V_{\mathrm{g}}(\mathrm{t})$ and $I_{\mathrm{g}}(\mathrm{t})$

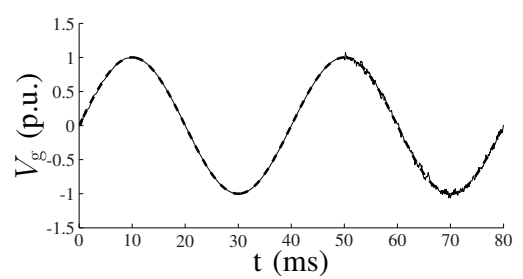

(c) Second reference load: $V_{\mathrm{g}}(\mathrm{t})$

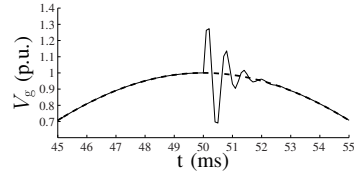

(b) Detail of $V_{\mathrm{g}}$ in (a)
Fig. 17. PIPD 1 - Cascade PI-PD control $\left(-=v_{\mathrm{g}}(\mathrm{t})\right.$ (p.u. $)$; --- $=v_{\mathrm{g}}^{\star}(\mathrm{t})$ (p.u.); -...- $=i_{\mathrm{g}}(\mathrm{t})$ (p.u.))

controller of the outer voltage control loop equals

$$
1.9823 \frac{z-0.81}{z-1}
$$

giving the controlled system $T_{s}=2.14 \mathrm{~ms}, \mathrm{PM}=40^{\circ}, \mathrm{GM}=$ $6 \mathrm{~dB}, \% \mathrm{OS}=40.6$ and $f_{b}=695 \mathrm{kHz}$ (open loop bandwidth). This controller is referred to as PIP 3.

The simulation results depicted in Fig. 18 show that because of the addition of $v_{\mathrm{g}}$ in the controller's output, a better transient behavior compared to PIPD 1 is obtained. This is analogous as in the direct control strategy. Again, the regime results are slightly less accurate.

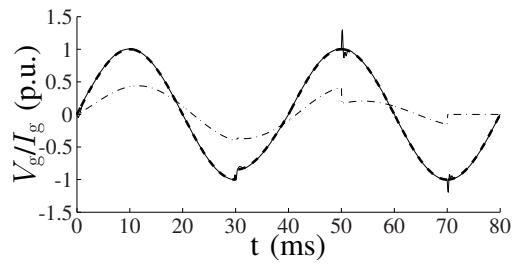

(a) First reference load: $V_{\mathrm{g}}(\mathrm{t})$ and $I_{\mathrm{g}}(\mathrm{t})$

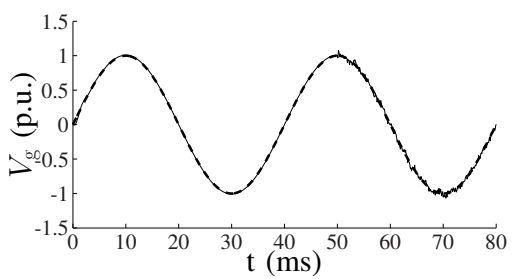

(c) Second reference load: $V_{\mathrm{g}}(\mathrm{t})$

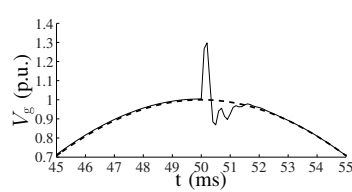

(b) Detail of $V_{\mathrm{g}}$ in (a)
Fig. 18. PIP 3 - Cascade PI-P control with forward compensation of $v_{\mathrm{g}}$ ($=v_{\mathrm{g}}(\mathrm{t})$ (p.u.); ---- $=v_{\mathrm{g}}^{\star}(\mathrm{t})$ (p.u.); -.-.-. $=i_{\mathrm{g}}(\mathrm{t})$ (p.u. $\left.)\right)$

\section{Conclusion: comparison of controllers}

The performance of all controllers is summarized in Table 1 In this table, the voltage error $e$ is given, which is defined as:

$$
e=\sqrt{\frac{1}{N} \sum_{\mathrm{i}=1}^{N}\left(v_{\mathrm{g}, \mathrm{i}}-v_{\mathrm{g}, \mathrm{i}}^{\star}\right)^{2}} .
$$


The parameter $N$ is defined such that the voltages in $39.9 \leq$ $t \leq 49.9 \mathrm{~ms}$ are compared. In this way, for the first reference load, the regime performance is compared and for the second one the parameter sensitivity.

TABLE I

STEADY-STATE PERFORMANCE CONTROLLERS

\begin{tabular}{|c|c|c|}
\hline & $e($ load 1) & $e$ (load 2) \\
\hline PID 1 & 0.0443 & unstable \\
PID 2 & 0.0520 & unstable \\
PID 3 & 0.1674 & 0.1160 \\
PIPD 1 & 0.0237 & 0.0258 \\
PIPD 2 & 0.0494 & 0.0404 \\
PIP 3 & 0.0944 & 0.0944 \\
\hline
\end{tabular}

The robustness to measurement noise of all controllers is similar.

Although, the PID 1 and 2 controllers have a good regime tracking, they are very sensitive to model faults of $L$. The PID 3 controller is less sensitive to these model faults, shows a better transient behavior, but a slightly less accurate steadystate performance compared to the other direct controllers.

In the cascade control, a good parameter robustness is obtained. The PIPD 1 and 2 controllers have similar results and achieve a slightly better steady-state tracking performance than PID 1 and 2. The transient results are similar, but as discussed above, their model robustness is a very important advantage. The PIP 3 controller has the advantage that the current control loop only requires a P-controller, achieves a very good transient response, but a slightly less accurate regime tracking compared to PIPD 1 and 2. Compared to PID 3 , its steady-state voltage tracking and model robustness are better.

To summarize, from these simulations, it follows that using cascade control of VSIs in islanded microgrids is possible, by adding current measurements in the control system. Cascade control is especially beneficial when considering model inaccuracies. Also, the regime error is lower in cascade control compared to direct control. When adding a grid voltage measurement to the output of the controller, both direct and cascade control show a better transient response and parameter sensitivity. Without the forwarding of this voltage measurement, either phase-lag compensation in the reference signal or adding the reference voltage to the controller's output is required for a good controllers performance.

\section{EXPERIMENTAL RESUlTS}

The previous theoretical and simulation results have been verified on an experimental set-up. A full bridge single phase converter is used with the same parameters as in the simulations. This converter was designed in the lab based on a Fuji IGBT Intelligent Power Module (IPM). A Freescale 56F8367 digital signal processor is used to implement the different controllers and generate the Pulse Width Modulated switching signals for the converter. Both direct and cascade controllers are digitally implemented with a differential equation and will now be discussed. The same load is used in all the experiments, which is an RL load (2.5 A, $\cos \phi=0.8)$. The figures in this section show the set point and measured grid voltage and the measured grid current. Also, the difference $e_{v}$ between the set point and measured grid voltages is shown.

\section{A. Direct control}

1) Without forward compensation: The PID 1 controller does not use forward compensation. Analogous as in the simulations, this leads to a phase-lag in a steady-state situation, which was compensated by a phase-lead in the reference signal in the experiments. Fig. 19 shows the measurement results.

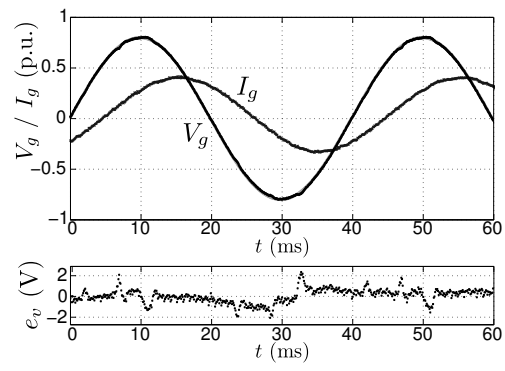

(a) $V_{\mathrm{g}}(\mathrm{t})$ and $e_{v}(\mathrm{t})$

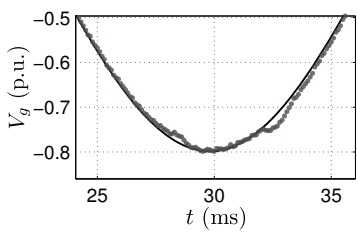

(b) Detail of $V_{\mathrm{g}}$

Fig. 19. PID 1 - Direct control without forward compensation of $v_{g}$ : Experiment, RL load $(2.5 \mathrm{~A}, \cos \phi=0.8)$

The measurement shows good steady-state tracking of the set point voltage, but an accurate phase-lag compensation was necessary to achieve this.

2) With forward compensation: The PID 3 controller uses forward compensation of the voltage measurement $v_{g}$. Therefore, the phase-lag of PID 1 is not longer present such that a phase-lag compensation is unnecessary. Fig. 20 shows a measurement of the PID 3 controller.

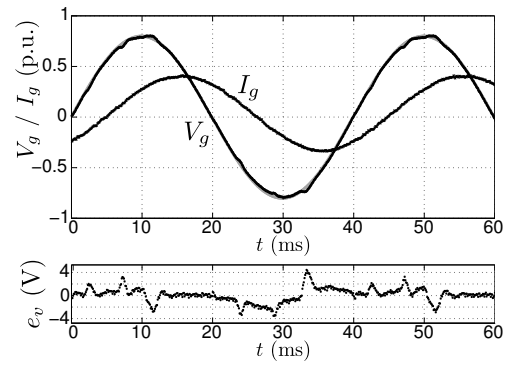

(a) $V_{\mathrm{g}}(\mathrm{t})$ and $e_{v}(\mathrm{t})$

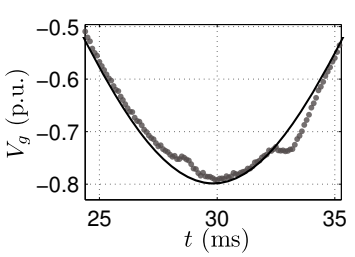

(b) Detail of $V_{g}$
Fig. 20. PID 3 - Direct control with forward compensation of $v_{g}$ : Experiment, RL load (2.5A, $\cos \phi=0.8)$

This figure clearly shows that a good steady-state tracking of the set point is maintained, although the phase-lag compensation was omitted. Thanks to the forward compensation, this satisfactory performance is achieved. Like in the simulations, a slightly better steady-state voltage tracking is obtained by the PID 1 controller compared to PID 3. However, noise and measurement inaccuracies qualify this conclusion.

\section{B. Cascade control}

1) Without forward compensation: The PIPD 1 controller does not use forward compensation. Hence, a phase lag com- 
pensation is needed to achieve a good steady state tracking. Fig. 21 shows a measurement of the PIPD 1 controller.

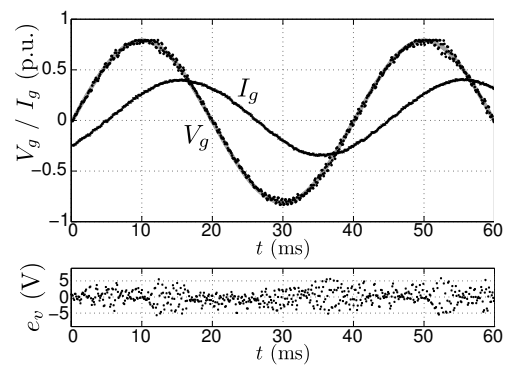

(a) $V_{\mathrm{g}}(\mathrm{t})$ and $e_{v}(\mathrm{t})$

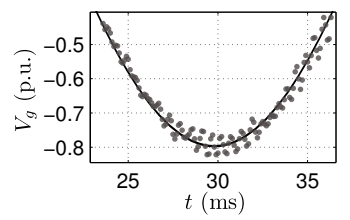

(b) Detail of $V_{\mathrm{g}}$
Fig. 21. PIPD 1 - Cascade PI-PD control:

Experiment, RL load $(2.5 \mathrm{~A}, \cos \phi=0.8)$

Again, good steady state tracking is achieved, but with the cost of including phase-lag compensation.

2) With forward compensation: The PIP 3 controller uses forward compensation of the measured voltage $v_{g}$, such that a phase-lag compensation becomes unnecessary. Also, the derivative action of the current controller was omitted. Fig. 22 shows the measurement results of the PIP 3 controller.

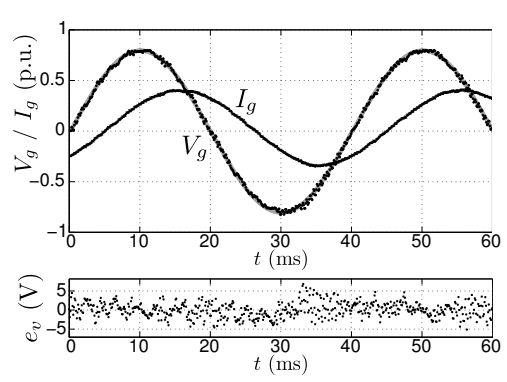

(a) $V_{\mathrm{g}}(\mathrm{t})$ and $e_{v}(\mathrm{t})$

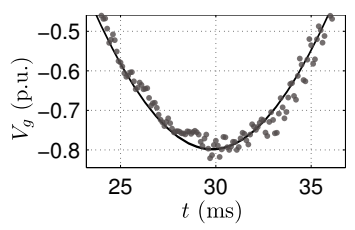

(b) Detail of $V_{\mathrm{g}}$
Fig. 22. PIP 3 - Cascade PI-P control with forward compensation of $v_{g}$ : Experiment, RL load $(2.5 \mathrm{~A}, \cos \phi=0.8)$

Although no phase compensation was applied, the PIP 3 controller has a good steady state tracking performance thanks to the forward compensation of $v_{g}$. Therefore, the result is similar to that of the PIPD 1 controller.

This shows that using cascade control is possible for voltage control in islanded microgrids. In the experiments, a good performance is obtained with all four controllers. Clearly, like in the simulations, either phase-lag compensation or forward voltage compensation is required to achieve a good tracking performance.

\section{CONCLUSIONS}

A theoretical analysis of voltage control in islanded microgrids shows that cascade control is possible and using measured current signals in this control scheme is beneficial for the overall system performance. Thanks to the $i_{\mathrm{g}}$ injection, the tuning of the controllers became independent of the load. This is a significant benefit from control point of view, since in practice the load is varying and not known. Also, for cascade control, a combined PI-P controller is sufficient, while direct control requires the usage of a PID controller for a stable operation

This is demonstrated by means of simulation as well as experimental results. In both the direct and the cascade control, phase-lag compensation is required to compensate for the phase-lag otherwise present in the regime voltage tracking. To avoid this, forward voltage compensation, thus adding a measurement of the grid voltage to the output of the controller, shows good results. By using these simple yet effective PIDtype controllers, accurate voltage control in islanded microgrids is obtained.

\section{REFERENCES}

[1] R. H. Lasseter, A. Akhil, C. Marnay, J. Stephens, J. Dagle, R. Guttromson, A. Meliopoulous, R. Yinger, and J. Eto, "The CERTS microgrid concept, white paper on integration of distributed energy resources," in California Energy Commission, Office of Power Technologies - U.S. Department of Energy, LBNL-50829, http://certs.lbl.gov, Apr. 2002.

[2] A. Engler, O. Osika, M. Barnes, N. Jenkins, and A. Arulampalam, DB1 Local Micro Source controller strategies and algorithms. www.microgrids.eu/micro2000, European Commission, Feb. 2004.

[3] H. Jiayi, J. Chuanwen, and X. Rong, "A review on distributed energy resources and microgrid," Renewable and Sustainable Energy Reviews, vol. 12 , no. 9, pp. 2472-2483, Dec. 2008.

[4] N. Lidula and A. D. Rajapakse, "Microgrids research: A review of experimental microgrids and test systems," Renewable and Sustainable Energy Reviews, vol. 15, no. 1, pp. 186-202, 2011.

[5] H. Farhangi, "The path of the smart grid," in IEEE Power \& Energy Magazine, Jan./Feb. 2010, vol. 8, no. 1, pp. 18-28.

[6] Q. Yang, J. A. Barria, and T. C. Green, "Communication infrastructures for distributed control of power distribution networks," IEEE Trans. Ind. Informat., vol. 7, no. 2, pp. 316-327, May 2011.

[7] P. Palensky and D. Dietrich, "Demand side management: Demand response, intelligent energy systems, and smart loads," IEEE Trans. Ind. Informat., vol. 7, no. 3, pp. 381-388, Aug. 2011.

[8] European Commission, European Technology Platform (ETP) Smart Grids. (2007) Strategic research agenda for Europe's electricity networks of the future.

[9] European Energy Research Alliance (EERA). (launched June 2010) Coordinating energy research for a low carbon Europe.

[10] R. H. Lasseter and P. Paigi, "Microgrid: A conceptual solution," in Proc. IEEE Power Electron. Spec. Conf. (PESC 2004), Aachen, Germany, 2004.

[11] M. Marwali, J.-W. Jung, and A. Keyhani, "Control of distributed generation systems - part II: Load sharing control," IEEE Trans. Power Electron., vol. 19, no. 6, pp. 1551-1561, Nov. 2004.

[12] F. Katiraei, R. Iravani, N. Hatziargyriou, and A. Dimeas, "Microgrids management: controls and operation aspects of microgrids," Power and Energy Magazine, vol. 6, no. 3, pp. 54-65, May/June 2008.

[13] C. Sao and P. Lehn, "Control and power management of converter fed microgrids," IEEE Trans. Power Syst., vol. 23, no. 3, Aug. 2008.

[14] J. M. Guerrero, J. Matas, L. García de Vicuña, M. Castilla, and J. Miret, "Decentralized control for parallel operation of distributed generation inverters using resistive output impedance," IEEE Trans. Ind. Electron., vol. 54, no. 2, pp. 994-1004, Apr. 2007.

[15] P. Arboleya, D. Diaz., J. M. Guerrero, P. Garcia, F. Briz, C. GonzalezMoran, and J. G. Aleisxandre, "An improved control scheme based in droop characteristic for microgrid converters," Electric Power Systems Research, vol. 80, no. 10, pp. 1215 - 1221, Oct. 2010.

[16] W. Yao, M. Chen, J. M. Guerrero, and Z.-M. Qian, "Design and analysis of the droop control method for parallel inverters considering the impact of the complex impedance on the power sharing," IEEE Trans. Ind. Electron., vol. 58, no. 2, pp. 576-588, Feb. 2011.

[17] J. M. Guerrero, J. C. Vásquez, J. Matas, L. García de Vicuña, and M. Castilla, "Hierarchical control of droop-controlled AC and DC microgrids - A general approach towards standardization," IEEE Trans. Ind. Electron., vol. 58, no. 1, pp. 158-172, Jan. 2011.

[18] H. J. Avelar, W. A. Parreira, J. B. Vieira, L. C. Gomes de Freitas, and E. A. A. Coelho, "A state equation model of a single-phase gridconnected inverter using a droop control scheme with extra phase shift control action," IEEE Trans. Ind. Electron., vol. 59, no. 3, pp. 15271537, Mar. 2012 
[19] T. L. Vandoorn, B. Meersman, L. Degroote, B. Renders, and L. Vandevelde, "A control strategy for islanded microgrids with dc-link voltage control," IEEE Trans. Power Del., vol. 26, no. 2, pp. 703-713, Apr. 2011.

[20] Y. Li and Y. W. Li, "Power management of inverter interfaced autonomous microgrid based on virtual frequency-voltage frame," IEEE Trans. on Smart Grid, vol. 2, no. 1, pp. 30-40, Mar. 2011.

[21] S. Dasgupta, S. N. Mohan, S. K. Sahoo, and S. K. Panda, "A plug and play operational approach for implementation of an autonomous-microgrid system," IEEE Trans. Ind. Informat., vol. 8, no. 3, Aug. 2012.

[22] N. Hatziargyriou, H. Asano, R. Iravani, and C. Marnay, "Microgrids: an overview of ongoing research, development, and demonstration projects," in IEEE Power \& Energy Magazine, Jul./Aug. 2007, vol. 5, no. 4, pp. 78-97.

[23] B. Kroposki, R. Lasseter, T. Ise, S. Morozumi, S. Papathanassiou, and N. Hatziargyriou, "A look at microgrid technologies and testing projects from around the world: making microgrids work," in IEEE Power \& Energy Magazine, May/June 2008, vol. 6, no. 3, pp. 40-53.

[24] European Commission, "European smartgrids technology platform: vision and strategy for Europe's electricity networks in the future," Community Research, Tech. Rep., 2006.

[25] T. C. Green and M. Prodanovíc, "Control of inverter-based micro-grids," Electric Power Systems Research, vol. 77, no. 9, pp. 1204-1213, July 2007.

[26] R. A. Mastromauro, M. Liserre, and A. Dell'Aquila, "Active damping of output LC filter resonance for vector-controlled VSI-fed AC motor drives," IEEE Trans. Ind. Informat., vol. 8, no. 2, pp. 241-254, May 2012.

[27] J.-W. Jung and A. Keyhani, "Control of a fuel cell based z-source converter," IEEE Trans. Energy Convers., vol. 22, no. 2, pp. 467-476, June 2007.

[28] N. A. Gounden, S. A. Peter, H. Nallandula, and S. Krithiga, "Fuzzy logic controller with MPPT using line-commutated inverter for threephase grid-connected photovoltaic systems," Renewable Energy, vol. 34, no. 3, pp. 909-915, Mar. 2009.

[29] A. Y. Sendjaja and V. Kariwala, "Decentralized control of solid oxide fuel cells," IEEE Trans. Ind. Informat., vol. 7, no. 2, pp. 163-170, May 2011.

[30] M. Ö. Efe, "Neural network assisted computationally simple $\mathrm{PI}^{\lambda} \mathrm{DI}^{\mu}$ control of a quadrotor UAV," IEEE Trans. Ind. Informat., vol. 7, no. 2, pp. 354-361, May 2011.

[31] Á. Cuenca, J. Salt, A. Sala, and R. Pizá, "A delay-dependent dual-rate pid controller over an ethernet network," IEEE Trans. Ind. Informat., vol. 7, no. 1, pp. 18-29, Feb. 2011.

[32] M. P. Kazmierkowski, M. Jasinski, and G. Wrona, "DSP-based control of grid-connected power converters operating under grid distortions," IEEE Trans. Ind. Informat., vol. 7, no. 2, pp. 204-2011, May 2011.

[33] N. Nise, Control System Engineering. Wiley, 6th edition, 2010, isbn 978-0470547564.

[34] M. Prodanović and T. C. Green, "High-quality power generation through distributed control of a power park microgrid," IEEE Trans. Ind. Electron., vol. 53, no. 5, pp. 1471-1482, Oct. 2006.

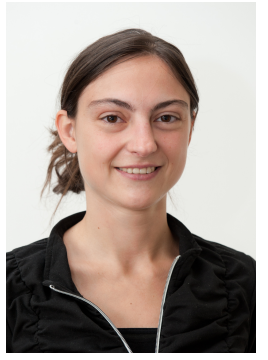

Tine Vandoorn (S'09) was born in Torhout, Belgium in 1985. She received the M.S. in electromechanical engineering from Ghent University, Ghent, Belgium, in 2008. In 2008, she joined the Electrical Energy Laboratory (EELAB) of Ghent University where she is currently pursuing the Ph.D. degree. Her present research interests include electric power systems, voltage and power control of distributed generation units, management of microgrids and smart microgrids. In 2009, she was awarded a grant as Ph.D. fellow of the Research Foundation - Flan-

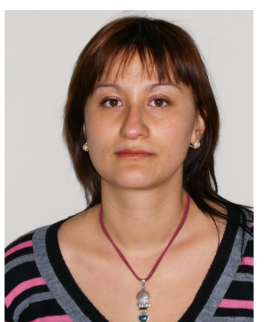

Clara M. Ionescu (S'06-M'09) was born in 1979, Cimpulung, Romania. She received the M.Sc. degree in industrial informatics and automation from "Dunarea de Jos" University, Galati, Romania, in 2003. She obtained the Ph.D. degree at Ghent University, Gent, Belgium in 2009, on identification of human respiratory system by means of fractional order models. Currently, she is a post-doc fellow in the same university, involved in several international projects, with both industrial and biomedical applications, for identification and control. Her main research interests include biomedical applications, with identification and advanced control objectives. C. Ionescu is the holder of the prestigious postdoctoral grant from Flanders Research Center (FWO).

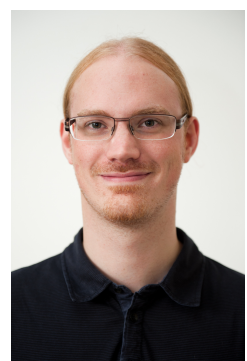

Jeroen De Kooning ( $\mathrm{S}^{\prime} 09$ ) was born in Kapellen, Belgium in 1987. He received the M.S. degree in electromechanical engineering from Ghent University, Belgium, in 2010. Since then, he is with the Electrical Energy Laboratory (EELAB) of Ghent University and is currently pursuing the Ph.D degree. His present research interests include wind energy systems and control of power electronic converters.

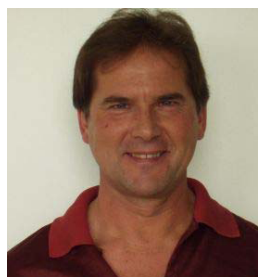

Robin De Keyser obtained the M.Sc. degree in electro-mechanical engineering in 1974 and the $\mathrm{PhD}$ degree in Control Engineering in 1980 from Ghent University, Belgium. He is currently full Professor of Control Engineering at the Faculty of Engineering, Ghent University. He is author/co-author of about 300 publications in journals, books, conference proceedings. He acted as external review expert in several European Commission research programs and is one of the pioneers who produced the original concepts of predictive control during the 1980's. His teaching and research activities include model predictive control, auto-tuning and adaptive control, modelling and simulation, system identification. The research is application-driven, with many pilot implementations in technical and non-technical systems, amongst others chemical, steel, marine, mechatronic, semiconductor, power electronics and biomedical.

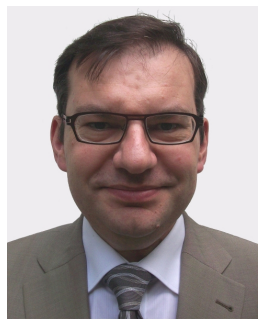

Lieven Vandevelde (M05 - SM07) was born in Eeklo, Belgium, in 1968. He graduated as Master of Electromechanical Engineering and received the $\mathrm{Ph} . \mathrm{D}$. degree from Ghent University, Belgium, in 1992 and 1997, respectively. He is with the Electrical Energy Laboratory (EELAB), Ghent University, where he has been a Professor in electrical power engineering since 2004. His research and teaching activities are in the field of electric power systems, electrical machines and (computational) electromagnetics. 